\title{
Review: spinal manipulative therapy is not better than standard treatments for low back pain
}

Assendelft WJ, Morton SC, Yu El, et al. Spinal manipulative therapy for low back pain. Cochrane Database Syst Rev 2004; (1):CD000447.

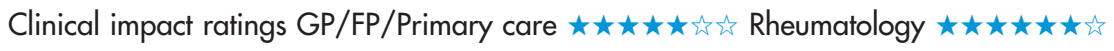

In patients with low back pain, is spinal manipulative therapy (SMT) effective for reducing pain and improving function?

\section{METHODS}

Data sources: Medline, CINAHL, EMBASE/Excerpta Medica, the
Cochrane Central Register of Controlled Trials (to 2000), and
reference lists.
$\begin{aligned} & \text { Study selection and assessment: randomised controlled trials } \\ & \text { (RCTs) that evaluated manipulation or mobilisation in patients } \\ & \text { with back pain. Cointerventions were allowed. } 2 \text { reviewers } \\ & \text { assessed the quality of study methods. }\end{aligned}$
Outcomes: pain (visual analogue or other scales) and functional
status (back pain specific scales).

\section{MAIN RESULTS}

39 studies (5486 patients) met the selection criteria. Acute low back pain $(<3$ wk duration). SMT led to short term improvement in pain when compared with sham therapy and to a modest short term improvement in pain when compared with a group of therapies judged to be ineffective or possibly harmful. SMT did not differ significantly from general practitioner (GP) care or analgesics, physical therapy or exercise, or back school (table). Chronic low back pain ( $>13$ wk duration). SMT led to improvements in pain (short and long term) and function (short term) relative to sham therapy. When compared with ineffective or harmful therapies, SMT led to short term improvement in function. SMT did not differ significantly from GP care or analgesics, physical therapy or exercise, or back school (table).

\section{CONCLUSION}

Spinal manipulative therapy is not better than standard treatments in patients with either acute or chronic low back pain.

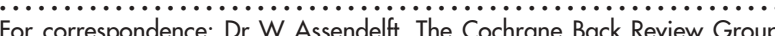
Toronto, Ontario, Canada. P.assendelff@nhg-nl.org

Source of funding: not stated.

\section{Commentary}

$\mathrm{N}$ otwithstanding the many previous reviews of RCTs of SMT for low back pain, this Cochrane review by Assendelft et al makes an important new contribution. By categorising the control interventions and constructing statistical models for main outcomes (pain and disability), the authors have succeeded in quantifying the strength of evidence for SMT compared with different alternative treatments. Their conclusions disagree with some, but not all, previous reviews. Data from 5486 participants in 39 trials are included. However, for some analyses, data were available from only 1 study. The magnitude of effect sizes and Cls presented is dependent upon both the underlying data and a back translation into clinically relevant values. The values used for this back translation have not been justified. This does not affect conclusions about statistical significance but could weaken inferences on the clinical importance of any differences.

No evidence of benefit was found for the key clinical question: "Is manipulation a useful addition to usual GP care?" However, the Cls are wide. For example, for long term function in chronic pain, the difference in Roland-Morris Disability Questionnaire (RMDQ) score is $0.8195 \% \mathrm{Cl}$ -4.6 to 6.1), much less than the 2.0 difference in RMDQ considered clinically important for individual patients. However, these data do not exclude the possibility that SMT has such a clinically important effect.

Additionally, even quite small clinical benefits from SMT could represent a cost effective addition to current services by reducing sick leave or the use of "ineffective or harmful therapies" and expensive secondary care services. This review shows that insufficient data exist to justify routine provision of SMT; it does not show that SMT is ineffective.

Because even quite small clinical benefits from SMT could be cost effective, there remains a need for further evidence to show whether SMT, when added to routine GP care, is either clinically or cost effective. Martin Underwood, MD Barts and The London London, UK

Spinal manipulation therapy (SMT) for low back pain*

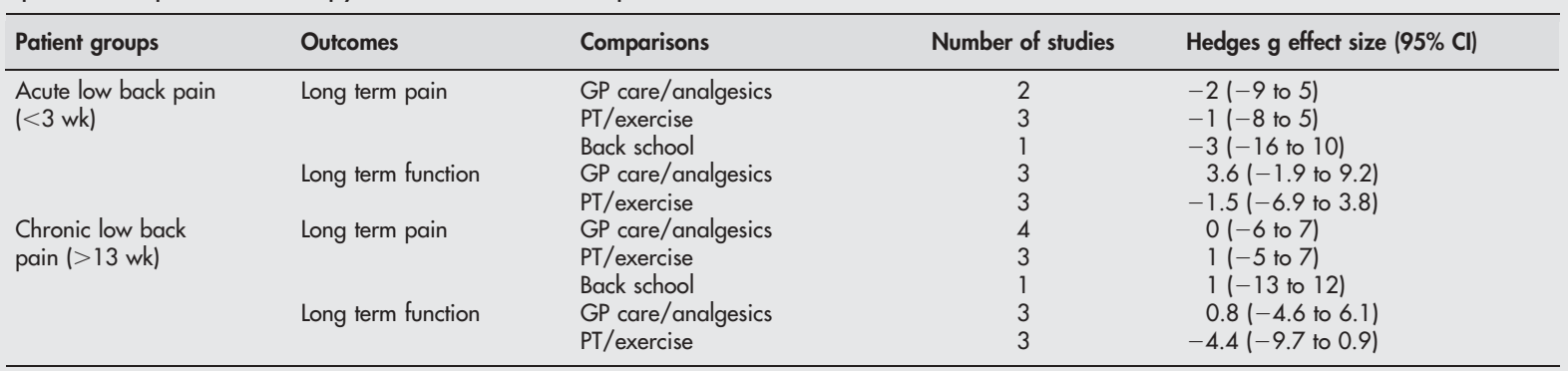

${ }^{*} \mathrm{GP}=$ general practice; PT = physical therapy. Effect sizes calculated using meta-regression. Positive effect sizes favour SMT. All effect sizes are statistically nonsignificant. 